\title{
The Anatomy of the Long Tail of Consumer Demand
}

\author{
Andrei Broder \\ Yahoo! Research, 701 First Ave., Sunnyvale, CA 94089, USA \\ broder@yahoo-inc.com
}

\begin{abstract}
The long tail of consumer demand is consistent with two fundamentally different theories. The first, and more popular hypothesis, is that a majority of consumers have similar tastes and only few have any interest in niche content; the second, is that everyone is a bit eccentric, consuming both popular and niche products. By examining extensive data on user preferences for movies, music, web search, and web browsing, we found overwhelming support for the latter theory. Our investigation suggests an additional factor in the success of "infiniteinventory" retailers such as Netflix and Amazon: besides the significant revenue obtained from tail sales, tail availability may boost head sales by offering consumers the convenience of "one-stop shopping" for both their mainstream and niche interests.

However, the observed taste eccentricity is much less than what is predicted by a fully random model whereby every consumer makes his product choices independent of each other and proportional to the product popularity. Hence, it appears consumers have a small a-priori propensity towards the popular or the exotic, but constructing a good model that agrees with the observed data as well as characterizing "eccentricity" are still open questions that we will discuss in some detail.

This talk is largely based on joint work with Sharad Goel, Evgeniy Gabrilovich, and Bo Pang: "Anatomy of the long tail: Ordinary people with extraordinary tastes", WSDM 2010.
\end{abstract}

